\title{
A Practical Method to Form Energy Efficient Connected $K$-Coverage in Wireless Sensor Networks
}

\author{
Zhen Jiang Robert Kline Jie Wu Fei Dai
}

\begin{abstract}
In the wireless sensor networks, an important issue of area coverage is to know how many sensor nodes are needed in a deployment under a certain kind of distribution. In that way, the nodes can be dense enough to form a complete and connected cover of interest area. Another issue that needs to be addressed is the "redundancy of connectivity" caused by the use of the well known sufficient condition that "the communication range $R$ is at least twice the sensing range $r$ ". In this paper, we show our result in solving these problems, a practical method to form a connected $k$-coverage with a connected dominating set (CDS). By introducing a measure of node density called the biggest vacant square territory (BVST), we prove that the point coverage offered by a CDS implies area coverage of the area of interest. The 1-point-coverage of a CDS with a reduced $R$ and a certain number of deploying sensors guarantees $k$-area-coverage, or simply $k$-coverage. Such assurance is verified via a simulation study on the CDS in [7] under random node distribution.
\end{abstract}

Keywords: $k$-coverage, connectivity, connected dominating set (CDS), energy efficiency, wireless sensor networks.

\section{INTRODUCTION}

Recent advances in micro-electromechanical systems, digital electronics, and wireless communications have enabled the development of low-cost, low-power, and multifunction sensor devices [5]. These devices can operate autonomously to gather, process, and communicate information about their environments. When a large number of devices collaborate using wireless communications and an asymmetric, many-to-one data flow, they constitute a wireless sensor network (or simply sensor network) [2]. Applications of sensor networks include battlefield surveillance, biological detection, home appliance, smart spaces, and inventory tracking [2], [6], [8], [12]. In a sensor network, each sensor is called a node. Each node's sensing area can be approximated as a disk around the node. Each node can measure or observe the physical parameter or event in its own sensing area and can use radio-frequency technology to communicate with other nodes in its vicinity, which is also called its communication area. The nodes inside the communication area are called its neighbors and two neighboring nodes are directly connected. For each node, its communication range $R$ is adjustable before the deployment

The work was supported in part by NSF grants ANI 0083836, CCR 9900646, CNS 0422762, CNS 0434533, and EIA 0130806. Dr. Zhen Jiang and Dr. Robert Kline are with Computer Science Department of West Chester University, West Chester, PA 19383. Dr. Jie Wu is with Computer Science and Engineering Department of Florida Atlantic University, Boca Raton, FL 33431. Dr. Fei Dai is with Electrical and Computer Engineering of North Dakota State University, Fargo, ND 58105. Contact E-mail zjiang@wcupa.edu. but its sensing range $r$ is always fixed. We assume all the nodes have the same ranges $R$ and $r$ after the deployment.

An important problem addressed in the literature is the area coverage problem [4], [11], [18]: How well do the nodes observe the physical space? In other words, how to have each location in the physical space of interest covered by at least one node (i.e., within the sensing area of at least one node [19]). As pointed out in [15], the area coverage, or simply coverage, is a measure of the quality of service (QoS) of the sensing function. For the purposes of accuracy of classification, tracking, and intrusion detection [3], [10], [25], some sensor networks require multiple coverage (i.e., each location covered by $k$ nodes [21]) rather than just 1-coverage. A complete connected $k$-coverage is achieved only if the nodes can k-cover the area of interest and remain connected. Without sufficient coverage, the network cannot guarantee the quality of sensing service. Without network connectivity, nodes may not be able to send data back to the remote base station.

To guarantee coverage, in most existing methods, it is assumed that the nodes are densely deployed and a node can always be found close to a certain location in the interest area within sensing range. For a certain deployment $D$ with total $M=|D|$ nodes deployed, the problem is how big this $M$ must be to provide a high confidence of ensuring a complete and connected coverage. This problem cannot be solved in any probability model [9], [13], [16] because a probability cannot represent the coverage situation when we pursue the complete coverage. We cannot guarantee the coverage of a position even when the probability is as high as $99.99 \%$. To guarantee the connectivity of coverage, most previous work is based on the sufficient condition that "the communication range $R$ is at least twice the sensing range $r "$ [19], [22], [24]. As the number of coverage required $k$ increases, the density of nodes deployed needs to increase and, respectively, more nodes will become neighboring nodes within the communication range. Many communications among those neighboring nodes are redundant for only sending the data of a single node. This issue is also called the "redundancy of connectivity".

In this paper, we present our results in solving these two problems. The contribution is threefold. First, we introduce a localized measure of sensing coverage, vacant square territory, and use the size of the biggest vacant square territory (BVST) as a character of the part of the sensor network covering the area of interest. Second, we prove the connectivity of that part of the network and the existence of its connected dominating set (CDS) given a certain communication range 


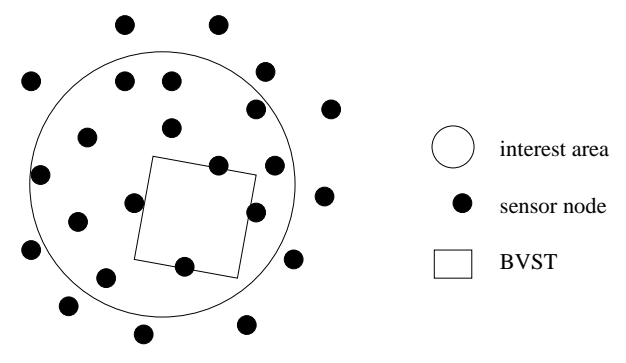

Fig. 1. An example of the biggest vacant square territory (BVST) in the area of interest.

$R$. We further prove that the point coverage of such a CDS provides a complete connected $k$-coverage. Third, based on this, a practical method applying CDS to form the required coverage is proposed. We study the size of BVST in random node distribution $D$ in the deployment. We conclude that with total $|D|$ nodes deployed, the minimum BVST $b$ derived from the simulation results ensures a complete connected $k$ coverage in real applications.

A short summary of our approach follows. First, we study the sensor networks where nodes are randomly deployed to cover the area of interest. The biggest vacant square territory (BVST) with the size of $b \times b$ in the area of interest that does not contain any node inside is found (see in Figure 1). After that, we prove the connectivity of the network in the area of interest and the existence of its CDS when $R>\sqrt{2} b$. Based on the properties of the CDS and the BVST, any square territory $Q$ with the size of $b \times b$ will contain at least one node and we can always find an active CDS node nearby around $Q$ within the communication range $R$. In other words, we can always find a CDS node within a $(b+2 R) \times(b+2 R)$ square territory. If the sensing range $r$ of this CDS node is longer than $\sqrt{2} b+R$, its sensing area can cover the area of $Q$, i.e., such a CDS node will provide 1-coverage to cover $Q$. Moreover, if the sensing range of CDS nodes in $k$ different $(b+2 R) \times(b+2 R)$ nearby squares is longer than their distance to any position in $Q$, those $k$ active nodes together will provide $k$-coverage to cover $Q$. Therefore, given enough nodes, the corresponding BVST size in the area of interest ensures that a connected $k$-coverage can be achieved simply by adjusting the communication range $R$. Our simulation results agree with the above analytical results.

Not just simply connecting all the localized covers [1], [18], our approach is to estimate the degree of coverage of the CDS. By reducing the communication range, our approach is to form a dense 1-point-coverage of CDS in such a way that the active nodes are close to each other to provide enough overlaps of area coverage. In this way, the connectivity redundancy can be cut down greatly while keeping a connected $k$-coverage. As we show in the later discussion, the use of CDS can reduce the communication range greatly for only keeping the necessary connectivity while providing the required multiple coverage. A CDS is formed by selecting a few active nodes from a large amount of deployed nodes. This selection of CDS not only reduces the energy consumption of the network, thereby extending the operational lifetime of the network, but also makes the active nodes distributed more evenly so that the

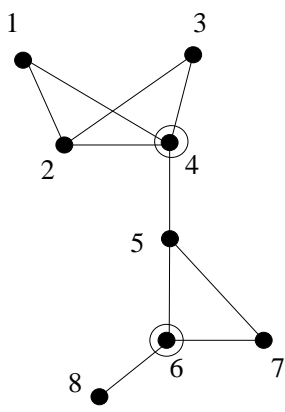

(a)

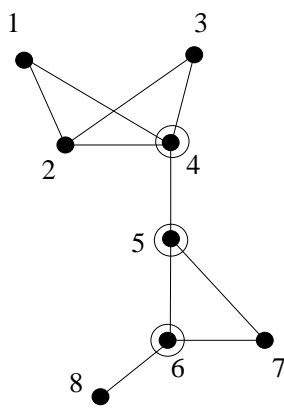

(b)
Fig. 2. Samples of the dominating set and the connected dominating set (The number represents the label of such a node).

effect of odd deployment distribution can be reduced to the minimum, and our estimate of the degree of coverage can be more accurate. It is noted that our approach does not need location information of node and it is distributed and localized.

The remainder of the paper is organized as follows: Section 2 introduces some necessary notations and preliminaries, including the formation of CDS and its dominating (also called 1-point-coverage) and connectivity properties. Section 3 discusses the impact of random node distribution and communication range $R$ on the quality of sensing service of a CDS. Based on this discussion, a practical approach to form connected $k$-coverage is proposed. Furthermore, we provide a sufficient condition, how many nodes are needed in the deployment for constructing such a CDS under the random node distribution, to ensure the required $k$-coverage. Section 4 presents the simulation results. Section 5 concludes this paper and provides ideas for future research.

\section{Preliminary}

With the assumption that all the sensors have the same communication range, a sensor network can be represented by a simple undirected graph $G=(V, E)$, where $V$ is a set of vertices (nodes) and $E$ is a set of undirected edges. An undirected edge $(u, v)$ denotes the connection between two neighboring nodes $u$ and $v$. The neighbor set $N(u)$ of node $u$ is defined as $\{w \mid(w, u) \in E\}$. We assume each node $u$ has been given a unique number label, $L(u)$. In the following discussion, we use $u$ and $L(u)$ alternatively to represent such a node. A set $V^{\prime} \subset V$ is a dominating set of $G$ if every node $v \in V-V^{\prime}$ is a neighbor of at least one node $u \in V^{\prime}$, i.e., 1-point-coverage. For example, both the set $\{4,6\}$ in Figure 2 (a) and the set $\{4,5,6\}$ in Figure 2 (b) are dominating sets. A set $V^{\prime}$ is connected if for any two nodes $u$ and $v \in V^{\prime}$, a path $\left(u, v_{1}\right),\left(v_{1}, v_{2}\right), \ldots,\left(v_{n}, v\right)\left(v_{i} \in V^{\prime}, 1 \leq i \leq n\right)$ in $E$ exists. A set $V^{\prime}$ is a connected dominating set (CDS) if it is dominating and connected. For example, the dominating set $\{4,5,6\}$ in Figure 2 (b) is a CDS.

The CDS can be constructed in either a global rippling algorithm [14] or a localized pruning algorithm [7]. The CDS nodes will keep active and the other nodes will be scheduled to sleep (i.e., inactive) status. However, the construction is based on a connected graph $G$. In the next section, we will relax 


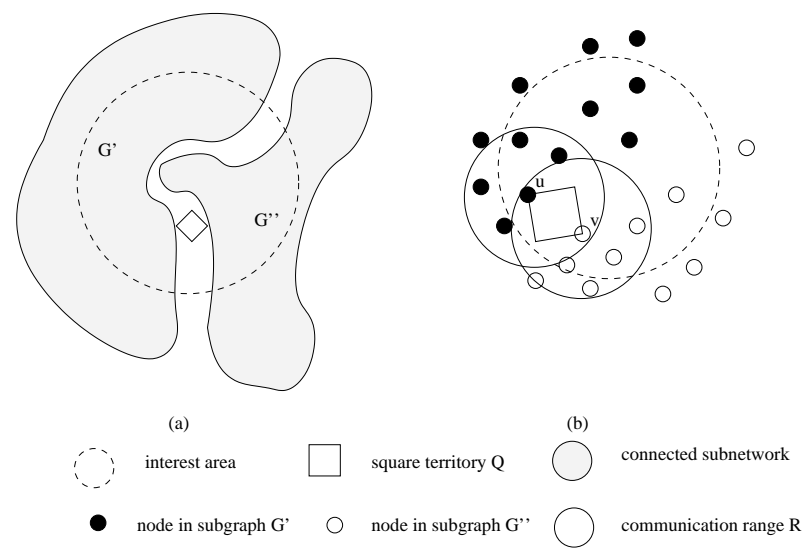

Fig. 3. The connectivity of CDS in the area of interest.

the connectivity assumption and ensure the existence of CDS covering the area of interest.

\section{Connected $k$-Coverage Sensor Network DERIVED FROM CDS}

In this section, we introduce a localized measure of sensing coverage, vacant square territory, and use the size of the biggest vacant square territory (BVST) as a character of the part of the sensor network covering the area of interest. Then, we discusses the relationships among communication range $R$, sensing range $r$, the edge of BVST $b$, and the degree of sensing coverage $k$. Based on this, an ensured deployment with enough number of nodes deployed is proposed to achieve required connected $k$-coverage under the random node distribution.

In a sensor network, the biggest vacant square territory (BVST) is defined as the biggest square territory that does not contain any node inside. The size of BVST is a measure of node density in the sensor networks. It is easy to see that a BVST must have nodes on at least three edges. Otherwise, the BVST can expand in both directions and become a bigger one.

The CDS can be derived from a connected graph $G$. With the information on the edges of the BVST in the sensor networks, we can adjust the communication range $R$ to ensure that $G$ is connected before we start the construction process (see in Figure 3). The following theorem becomes the fundamental block of building a CDS in sensor networks.

Theorem 1 (Existence of CDS): Assume the edge of the BVST in the area of interest of a sensor network is $b$. All the nodes in this area of interest will be connected if their communication range $R$ is larger than $\sqrt{2} b$.

Proof: If the nodes in the area of interest are not connected as a subnetwork, we can always find one connected subnetwork $G^{\prime}$ and the other part $G-G^{\prime}$ in such an area. The distance from a node in one subnetwork to another subnetwork is defined as the minimum distance from this node to any node in the second network. The distance between two subnetworks is defined as the minimum distance from any node of the first network to the second network. Assume $G^{\prime \prime}$ is the closest connected

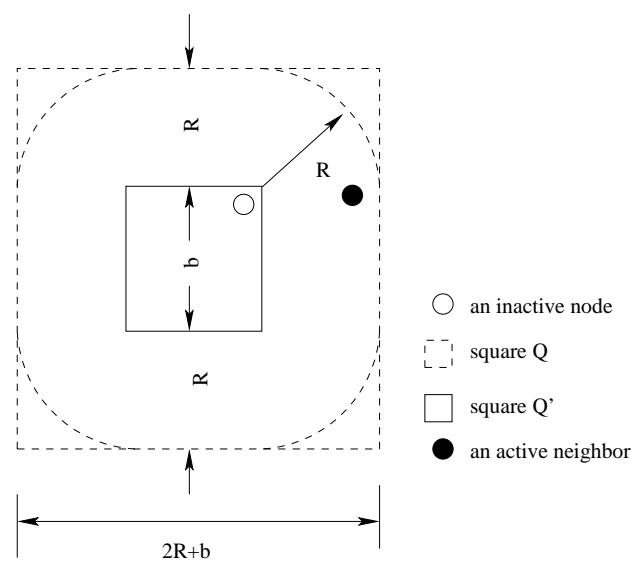

Fig. 4. Assurance of the existence of an active node (Lemma 1).

subnetwork to $G^{\prime}$ in $G-G^{\prime}$ in the area of interest. Assume node $u$ is the node in $G^{\prime}$ that has the minimum distance to $G^{\prime \prime}, \delta$. Assume node $v$ is such a node in $G^{\prime \prime}$ that its distance to $u$ is this $\delta$. Denote $Q$ as the square with opposite corners $u$ and $v$. A node in $G^{\prime}$ cannot appear in $Q$ since the distance between such a node and $v$ will be shorter than $\delta$. Neither can a node in $G^{\prime \prime}$ appear in $Q$, because such a node would make the distance from $u$ to $G^{\prime \prime}$ shorter. If there is a node of another subnetwork $G^{\prime \prime \prime}$ in $Q, G^{\prime \prime}$ will not be the closest subnetwork to $G^{\prime}$, which causes a contradiction. Therefore, $Q$ does not contain any node and $\delta \leq \sqrt{2} b$, based on the definition of BVST. When $R>\sqrt{2} b, G^{\prime}$ and $G^{\prime \prime}$ are connected because $u$ and $v$ can be connected, which causes a contradiction. In this way, it can be proved that all the subnetworks in the area are connected, i.e., all the nodes in the area are connected.

We have shown that the BVST has at least two nodes on different edges. Because of this, any BVST-size square area, including the edges, should contain at least one node. This ensures the existence of a node in a certain area in the original network $G$. Thus, we provide the following lemma to ensure the existence of an active CDS node in a certain area.

Lemma 1: If the edge of the BVST in the area of interest of a sensor network is $b$ and the communication range of all the nodes is $R$, a CDS has at least one active node in any square territory with the size $(b+2 R) \times(b+2 R)$.

Proof: In any square territory $Q$ with the size $(b+2 R) \times$ $(b+2 R)$, consider the central area $Q^{\prime}$ with the size of BVST $(b \times b) . Q^{\prime}$, including its edges, contains at least one node. After the construction of CDS, because it is dominating, we can always find an active node within the communication range $R$. Therefore, inside this $Q$, we can always find an active node.

Based on the above discussion on the existence of active node (CDS node), we can ensure the sensing coverage of a certain area from a nearby active node with sufficiently large sensing range $r$ (i.e., small $R$ ) in the following statement.

Theorem 2 ( $k$-coverage assurance): In the area of interest of a sensor network with communication range $R$, sensing range 


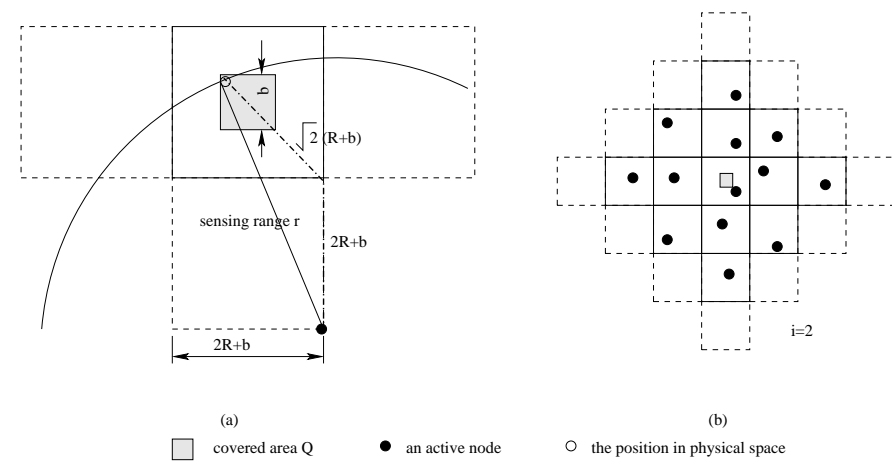

Fig. 5. Assurance of $k$-coverage.

$r$, and edge of BVST $b$, any position is covered by at least one active node (CDS node) if $r \geq \sqrt{2} b+R$, and by at least $k$ active nodes (CDS nodes) where $k=1+1 * 4+2 * 4+$ $\ldots+i * 4=1+2 * i *(i+1)>1$ such that $i=\max \{n \mid r>$ $n(2 R+b)+\sqrt{2}(b+R)\}$.

Proof: First, we prove that any BVST-size square territory $Q$ will be covered by at least one active node if $r \geq \sqrt{2} b+R$. For such square $Q$, we can always find a node $u$ and a nearby active node within communication range $R$ (because of the 1-point-coverage of CDS). The distance between any position in $Q$ and node $u$ is no more than $\sqrt{2} b$ since the size of $Q$ is $b \times b$. Therefore, the maximum distance between this position and that active node is less than $\sqrt{2} b+R$. In this way, the entire area of $Q$ is covered by that active node if $r \geq \sqrt{2} b+R$.

Then, for each square $Q$ with BVST-size, it is very easy to find $k=1+1 * 4+2 * 4+\ldots+i * 4$ nearby $(b+2 R) \times(b+2 R)$ size square territories (see Figure $5(\mathrm{~b})$ ). The distance from any node in $Q$ to a node in the farthest $(b+2 R) \times(b+2 R)$ size square is less than $i(2 R+b)+\sqrt{2}(b+R)$ (see Figure 5 (a)). Therefore, based on Lemma 1, any position in $Q$ will be covered by $k$ active nodes in those $(b+2 R) \times(b+2 R)$-size square territories.

Theorem 2 ensures the complete coverage of the area of interest by CDS nodes with sufficiently large sensing range $r$ (i.e., small communication range $R$ ). Moreover, if we can ensure the existence of a few active nodes within the sensing range from a position, all these active nodes will cover this position and provide multiple coverage. For simplicity, we assume the deployment area is larger than the area of interest so as to provide the same degree of coverage in the border area.

For example, when the edge of the BVST is no more than $1.2125 \mathrm{~m}$ and the sensing range $r$ is $10 \mathrm{~m}$, the communication range should be at least $1.7147 \mathrm{~m}$ so that a connected network graph can be ensured for the construction of CDS. If all the nodes adjust their communication range to $1.7147 m$, a 5 coverage can be ensured since $2(2 R+b)+\sqrt{2}(b+R)>$ $13.4234>r=10>0.7816>1(2 R+b)+\sqrt{2}(b+R)$.

Lemma 2: In CDS sensor networks with sensing range $r$, $R<\frac{\sqrt{2}}{2+\sqrt{2}+(1+2 \sqrt{2}) i} r$ and $b<\frac{\sqrt{2}}{2} R$ will ensure connected $k$-coverage, where $k=1+2 * i *(i+1)>1$.
Proof: If $R<\frac{\sqrt{2}}{2+\sqrt{2}+(1+2 \sqrt{2}) i} r$, we have

$$
\begin{array}{r}
\frac{r-(\sqrt{2}+2 i) R}{i+\sqrt{2}} \\
\frac{\frac{2+\sqrt{2}+(1+2 \sqrt{2}) i}{\sqrt{2}} R-(\sqrt{2}+2 i) R}{i+\sqrt{2}} \\
R \frac{\sqrt{2}+\frac{\sqrt{2}}{\sqrt{2}}+\left(\frac{1}{\sqrt{2}}+2\right) i-\sqrt{2}-2 i}{i+\sqrt{2}}= \\
R \frac{\frac{1}{\sqrt{2}} i+\frac{\sqrt{2}}{\sqrt{2}}}{i+\sqrt{2}}=\frac{R}{\sqrt{2}}>b
\end{array}
$$

Therefore, $r>(\sqrt{2}+2 i) R+(\sqrt{2}+i) b=i(2 R+b)+$ $\sqrt{2}(b+R)$, which ensures connected $k$-coverage where $k=$ $1+1 * 4+2 * 4+\ldots+i * 4=1+2 * i *(i+1)$ (based on Theorem 2).

From the result of Lemma 2, the nodes with sensing range $r=10 \mathrm{~m}$, the communication range $R=1.952 \mathrm{~m}$, and deployment which leads to $b<1.38 m$ will ensure connected 5 -coverage $(i=1)$. To ensure connected 10-coverage where $i=2$, set $R$ to $1.277 m$ and keep $b<0.9032 m$.

Theorem 3 ( $k$-coverage under node distribution $D$ ): In $C D S$ sensor networks with sensing range $r$, once we know the distribution function $D(b)$ which ensures the length of the edge of BVST is less than $b$, the communication range $R<\frac{\sqrt{2}}{2+\sqrt{2}+(1+2 \sqrt{2})} r$ and the node distribution $D\left(\frac{\sqrt{2}}{2} R\right)$ will ensure connected $k$-coverage, where $k=1+2 * i *(i+1)>1$.

Proof: Node distribution $D\left(\frac{\sqrt{2}}{2} R\right)$ leads to $b<\frac{\sqrt{2}}{2} R$. It is easy to prove the rest of the statement based on the results in Lemma 2.

When the required degree of coverage increases, the communication range and the corresponding size of BVST will be reduced. A small planned BVST-size $(b \times b)$ requires a high node density. It leads to a high expense cost if too many sensors are needed. The use of Theorem 3 will guarantee a cost-effective covering plan. First, calculate the maximum communication range $R$ and the maximum size of BVST (b) allowed (see in Theorem 2). After that, $\left|D\left(\frac{\sqrt{2}}{2} R\right)\right|$, the total number of nodes deployed under distribution $D\left(\frac{\sqrt{2}}{2} R\right)$ is calculated, where $D(b)$ ensures the length of the edge of BVST is less than $b$ (see in Figure 6 (b)). If the number of nodes required is too big, the covering plan will be too expensive to operate. For financial reasons, such a plan will fall through. Then, distribute the sensors to a certain level that ensures no vacant square space will be bigger than $b \times b$. After that, the CDS will guarantee the degree of coverage. The details are shown in Algorithm 1.

For example, if $r=10 \mathrm{~m}$ and $k=5, R=1.952 \mathrm{~m}$ $<\frac{\sqrt{2}}{2+\sqrt{2}+(1+2 \sqrt{2}) i} r$ and $\frac{\sqrt{2}}{2} R=1.380 \mathrm{~m}$. For the deployment that distributes all the nodes randomly in a $100 \mathrm{~m} \times 100 \mathrm{~m}$ square area, if we know that the BVST size will be no more than $1.380 \mathrm{~m}$ when at least 20,000 sensors are deployed (| $\left.D\left(\frac{\sqrt{2}}{2} R\right) \quad \mid=20,000\right)$ and if these 20,000 sensors are within the budget, connected 5-coverage for an interest area inside this square area will be successfully constructed and 
Algorithm 1: Construction of ensured connected $k$-coverage by using the sensors with the fixed sensing range $r$ ( $L$ is the maximum number of sensors allowed within the budget under node distribution $D$ ).

1) Based on Theorem 2, calculate the maximum communication range $R$ and the maximum size of BVST allowed $b<\frac{R}{\sqrt{2}}$. (a) If $k=1, R<r-\sqrt{2} b<\frac{r}{2}$, and (b) if $k>1, R<\frac{\sqrt{2}}{2+\sqrt{2}+(1+2 \sqrt{2}) i} r$ where $k \leq$ $1+1 * 4+2 * 4+\ldots+i * 4=1+2 * i *(i+1)$.

2) Calculate the total number of nodes needed for the distribution $D\left(\frac{\sqrt{2}}{2} R\right)$ where distribution $D(b)$ ensures the vacant square space is less than $b \times b, M=1$ $D\left(\frac{\sqrt{2}}{2} R\right) \mid$. If $M>L$, the covering plan is too expensive to operate and it will fall through.

3) Distribute the sensors under distribution $D\left(\frac{\sqrt{2}}{2} R\right)$ so that no vacant square space will be bigger than $b \times b$.

4) Apply the construction process of CDS, using either a global rippling algorithm [14] or a localized pruning algorithm [7].

5) The marked nodes (active nodes) are assured to provide connected $k$-coverage.

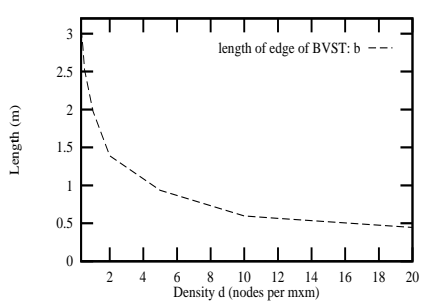

(a)

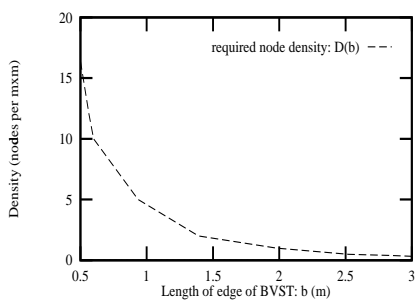

(b)
Fig. 6. Relationship between average node density $d$ and upper bound of the edge of BVST $b$.

such a degree of coverage can be guaranteed. If $k=42$, $R=0.627 m<\frac{\sqrt{2}}{2+\sqrt{2}+(1+2 \sqrt{2}) i} r$ and $\frac{\sqrt{2}}{2} R=0.4434 m$. To guarantee the maximum BVST-size $0.4434 \times 0.4434,225,000$ nodes are required to deploy. If the budget only allows for 200,000 sensors, this 42 -coverage cannot be guaranteed within the budget and such a coverage plan will fall through.

\section{SIMULATION}

In this section, we verify the above analytical results of coverage by using the experimental results from a simulator. The simulation is conducted to test if the coverage can be achieved as promised under the random distribution like throwing sensors from an airplane. It randomly allocates a given number of nodes in a $50 \mathrm{~m} \times 50 \mathrm{~m} 2$ 2-D free space to cover the area of interest inside.

First, we study the vacant square space right next to each node in the north in up to 10,000 samples for each case of average density (number of nodes / 2,500 square meters). When BVST for each case happens to be one of these squares, its size can be found in our simulator. Figure 6 (a) shows the results, which suggest that given enough nodes to increase

\begin{tabular}{|l|l|r|r|r|}
\hline \hline $\begin{array}{l}k, \# \text { of } \\
\text { coverage } \\
\text { expected }\end{array}$ & $R(m)$ & $b(m)$ & $\begin{array}{r}M, \text { total } \\
\text { number } \\
\text { of nodes }\end{array}$ & $\begin{array}{r}k^{\prime}, \text { expe- } \\
\text { rimental } \\
\text { results }\end{array}$ \\
\hline 1 & 5 & 3.535 & 625 & 2 \\
\hline $2-5$ & 1.952 & 1.380 & 5,000 & 27 \\
\hline $6-13$ & 1.277 & 0.903 & 13,750 & 78 \\
\hline $14-25$ & 0.949 & 0.671 & 22,500 & 140 \\
\hline $26-41$ & 0.755 & 0.533 & 36,250 & 225 \\
\hline \hline
\end{tabular}

TABLE I

ANALYTICAL AND EXPERIMENTAL RESULTS

the average node density to a certain level, the BVST in the networks can be ensured within a certain size. For example, the upper bound of the size of BVST is no larger than 1.5 meter $\times 1.5$ meter when we put 5,000 nodes and the average density reaches to 2 nodes/square-meter. This assurance can be seen in Figure 6 (b), where the node density ranges from 0.3 node/square-meter to 20 nodes/square-meter [17].

In our randomly deployed networks, the radius of sensing range is set to be 10 meters [20], [23], [24] for each node. Before the deployment, for the required degree of coverage, we can determine the communication range $R$ and the required size of $b$ to ensure the success of construction of CDS. Table I shows our analytical results of the required $R$ and $b$ (in Lemma 2) for the expected $k$ coverage (ranging from 1coverage to 41-coverage) under random node distribution for the $50 \mathrm{~m} \times 50 \mathrm{~m}$ area of interest. To ensure that the size of BVST will not be bigger than $b \times b$, the total number of nodes needed $M$ is determined based on Theorem 3 and the results in Figure 6 (b) (i.e., $M=|D(b)|$ ). The analytical results of this $M$ are also shown in Table I for a $k$-coverage ranging from 1 to 41 . For expected $k$ coverage in this 50 meter $\times 50$ meter area, we set up the required communication range $R$ and then position as many as $M$ nodes. After that, the CDS is constructed by using the pruning algorithm in [7]. To get the experimental results of minimum coverage of this CDS in the area of interest, many detection points (up to $100 \times 100$ per square meter) are allocated in the central area of this 50 meter $\times 50$ meter deployment area. At each detection point, we test the number of CDS nodes within the sensing range, i.e., the number of CDS nodes which can cover this position. It is not necessary to test the area close to the edge of the deployment area since, in most real applications, the area of interest will be smaller than the deployment area and is expected to locate in the central part of the latter one. The experimental results of minimum coverage tested are shown in Table I as $k^{\prime}$ and verify the correctness of our new protocol when no more than 50,000 nodes are allowed in the deployment.

The experimental results of minimum coverage in Table I show that the expected $k$-coverage can be achieved in the CDS in [7] when $R<r$. It is noted that the CDS construction in our simulator using pruning algorithm is the simplest one but not optimal. Up to $24 \%$ nodes will still remain active in the sensor networks; that is, coverage redundancy may exist. Our protocol can adopt any other CDS while holding the properties of CDS. In other words, our analytical results will be applicable for any sensor network where all the active nodes form a CDS. 


\section{Conclusion}

In summary, we proved that the point coverage of CDS in a wireless sensor network implies an area coverage for the entire area of interest. The connectivity assumption for the existence of CDS can be relaxed by adjusting the communication range based on the information of the size of BVST. By such adjustment, our approach processes in a conservative manner, such that each node in the area of interest and its nearby BVST-size area will be covered by at least $k$ CDS nodes $(k \geq$ 1). In a sensor network under the random node distribution, we provide enough deploying nodes to control the size of BVST, furthermore to ensure the existence of CDS and the corresponding connected $k$-coverage. Our protocol can support any CDS so long as it keeps the properties of connectivity and dominating. As future work, we plan to refine our connected $k$ coverage protocol by considering the irregular area of interest with "holes".

\section{REFERENCES}

[1] Z. Abrams, A. Goel, and S. Plotkin. Set k-cover algorithms for energy efficient monitoring in wireless sensor networks. Proc. of Information Processing in Sensor Networks. 2004.

[2] I. Akyildiz, W. Su, Y. Sankarasubramaniam, and E. Cayirci. Wireless sensor networks: A survey. Computer Networks. Vol. 38, 2002, pp. 393-422.

[3] A. Arora, P. Dutta, S. Bapat, V. Kulathumani, H. Zhang, V. Naik, V. Mittal, H. Cao, M. Gouda, Y. Choi, T. Herman, S. Kulkarni, U. Arumugarn, M. Nesterenko, A. Vora, and M. Miyashita. Line in the sand: A wireless sensor network for target detection, classification, and tracking. Technical report. OSU-CISRC-12/03-TR71, Ohio State University, 2003.

[4] M. Cardei and J. Wu. Energy-efficient coverage problems in wireless adhoc sensor networks. Journal of Computer Communications on Sensor Networks. 2005.

[5] J. Carle and D. Simplot-Ryl. Energy-efficient area monitoring for sensor networks. Computer. Vol. 37, Issue 2, Feb., 2004, pp. 40-46.

[6] A. Cerpa, J. Elson, M. Hamilton, J. Zhao, D. Estrin, and L. Girod. Habitat monitoring: Application driver for wireless communications technology. Proc. of ACM SIGCOMM Workshop on Data Communications in Latin America and the Caribbean. 2001, pp. 3-5.

[7] F. Dai and J. Wu. A generic distributed broadcast scheme in ad-hoc wireless networks. IEEE Transactions on Computers. Vol. 53, No. 10, Oct. 2004, pp. 1343-1354.

[8] D. Estrin, R. Govindan, J. Heidemann, and S. Kumar. Next century challenges: Scalable coordination in sensor networks. Proc. of ACM International Conference on Mobile Computing and Networking (MOBICOM'99). 1999, pp. 263-270.

[9] P. Gupta and P. Kumar. Critical power for asymptotic connectivity in wireless networks. in Stochastic Analysis, Control, Optimization and Applications: A Volume in Hornor of W.H. Fleming. Birkhauser, 1998, pp. 547-566, also available at http://www.ee.surrey.ac.uk/Personal/ G.Aggelou/PAPERS/connectivity.pdf.

[10] D. Hall and J. Llinas. Handbook of multisensor data fusion. CRC Press. 2001.

[11] C. Huang and Y. Tseng. The coverage problem in a wireless sensor network. Proc. of ACM Int'l Workshop on Wireless Sensor Networks and Applications (WSNA). 2003, pp. 115-121.

[12] J. Kahn, R. Katz, and K. Pister. Next century challenges: Mobile networking for smart dust. Proc. of ACM International Conference on Mobile Computing and Networking (MOBICOM'99). 1999, pp. 271-278.
[13] S. Kumar, T. Lai, and J. Balogh. On $k$-coverage in a mostly sleeping sensor network. Proc. of ACM International Conference on Mobile Computing and Networking (MOBICOM'04). 2004, pp. 144-158.

[14] T. Lin, S. Midkiff, and J. Park. Minimal connected dominating set algorithms and application for a MANET routing protocol. Proc. of the IEEE International Conference on Performance, Computing, and Communications. April 2003, pp. 157-164.

[15] S. Meguerdichian, F. Koushanfa, M. Potkonjak, and M. Srivastava. Coverage problems in wireless ad-hoc sensor networks. Proc. of IEEE Infocom. 2001, pp. 1380-1387.

[16] S. Shakkottai, R. Srikant, and N. Shroff. Unreliable sensor grids: coverage, connectivity and diameter. Proc. of the IEEE INFOCOM'03. 2003, pp. 1073-1083.

[17] E. Shih, S. Cho, N. Lckes, R. Min, A. Sinha, A. Wang, and A. Chandrakasan. Physical layer driven protocol and algorithm design for energy-efficient wireless sensor networks. Proc. of ACM Special Interest Group on Mobile of Systems Users, Data, and Computing (SIGMOBILE'01). 2001, pp. 272-286.

[18] S. Slijepcevic and M. Potkonjak. Power efficient organization of wireless sensor networks. Proc. of IEEE International Conference on Communications. 2001, pp. 472-476.

[19] D. Tian and N. Georganas. Connectivity maintenance and coverage preservation in wireless sensor networks. Ad Hoc Networks Journal. 2005, pp. 744-761, available at http://www.discover.uottawa.ca/ publications/adhoc_paper.pdf.

[20] D. Tian and N. Georganas. A coverage-preserving node scheduling scheme for large wireless sensor networks. Proc. of the 1st ACM Workshop on Wireless Sensor Networks and Applications. 2002.

[21] P. Varshney. Distributed detection and data fusion. Spinger-Verlag. New York, 1996.

[22] X. Wang, G. Xing, Y. Zhang, C. Lu, R. Pless, and C. Gill. Integrated coverage and connectivity configuration in wireless sensor networks. Proc. of the 1st ACM Conference on Embedded Networked Sensor Systems. 2003.

[23] F. Ye, G. Zhong, J. Cheng, S. Lu, and L. Zhang. PEAS: A robust energy conserving protocol for long-lived sensor networks. Proc. of the 23rd International Conference on Distributed Computing Systems (ICDCS'03). 2003, pp. 28-37.

[24] H. Zhang and J. Hou. Maintaining sensing coverage and connectivity in large sensor networks. Technical Report UIUC. UIUCDCS-R-20032351, 2003.

[25] J. Zhao and R. Govindan. Understanding packet delivery performance in dense wireless sensor networks. Proc. of ACM Sensys. 2003, pp. $1-13$. 